\title{
La mercantilización de la comunidad. Problemas éticos del turismo comunitario
}

\section{The Commodification of the Community. Ethical Problems of Community-Based Tourism}

\author{
MARCOS ALONSO FERNÁNDEZ (Universidad Adolfo Ibáńez) y ELIZABETH DE LA CRUZ \\ (Universidad de Otavalo)
}

Artículo recibido: 15 de marzo de 2020 Solicitud de revisión: 3 de mayo de 2020 Artículo aceptado: 8 de octubre de 2020

Alonso, Marcos y de la Cruz, Elizabeth (2021). La mercantilización de la comunidad. Problemas éticos del turismo comunitario. Recerca. Revista de Pensament i Análisi, 26(1), pp. 93-110. doi: http://dx.doi.org/10.6035/Recerca.2021.26.1.5

Resumen

El objetivo de la presente investigación es analizar algunos problemas éticos del turismo comunitario (TC). El TC es una forma novedosa de turismo que ha aparecido en las últimas décadas en diferentes lugares del mundo, especialmente en aquellas zonas de importante población indígena. Las investigaciones hasta ahora han destacado mayoritariamente las virtudes de este tipo de turismo, y las pocas críticas que han aparecido contra el TC han tenido que ver con fallas en su funcionamiento o en el beneficio que en ocasiones no llegaba para las comunidades. Si bien las complicaciones éticas asociadas al TC no han pasado desapercibidas, en este artículo abordaremos algunos problemas éticos del TC a los que no se ha prestado tanta atención hasta el momento - como aquellos relacionados con el campo de las indigenous ethics-, que demandan una profunda reflexión sobre la utilización comercial de las comunidades.

Palabras clave: turismo comunitario, ética, éticas indígenas, mercantilización.

Abstract

The objective of this research is to analyse the ethical problems of Community-Based Tourism (CBT). CBT is a novel form of tourism that has appeared in recent decades in different parts of the world, especially in areas with large indigenous populations. The investigations until now have primarily highlighted the virtues of this type of tourism, and the few criticisms that have appeared against СBT were related to its functional shortcomings and the benefits that sometimes do not reach the communities. While the ethical complications 
associated with the CBT have not gone unnoticed, in this article we address some ethical problems of the CBT that have not received as much attention so far - such as those related to the field of indigenous ethics-, that demand a deep reflection on the commercial use of communities.

Key Words: Community-Based Tourism, ethics, indigenous ethics, commodification.

\section{INTRODUCCIÓN}

El turismo es uno de los grandes pilares económicos del mundo contemporáneo. Para un gran número de países, el turismo es uno de los principales motores generadores de desarrollo socioeconómico y empleo. Durante las últimas décadas, el turismo se presentó como una estrategia económica infalible, en la que se generaban grandes beneficios sin necesidad de invertir excesivamente, puesto que en muchas ocasiones el lugar mismo, con sus paisajes, monumentos y valores culturales, era el principal atractivo (Burns y Holden, 1995; Mowforth y Munt, 1998; Vargas-Cumbajín et al., 2017). Con el paso del tiempo se han empezado a demandar nuevas formas de turismo, en las cuales se prima el sentido de experiencia auténtica y la pequeña escala (Hampton, 2003).

En este contexto se enmarca el surgimiento del turismo comunitario (en adelante, TC). El TC estuvo ligado en sus inicios al etnoturismo y al ecoturismo (Fennell, 2008), pero pronto fue tomando un camino propio y diferenciado. En 1985 la Asamblea General de la Organización Mundial del Turismo (OMT, 1985), en la Declaración de Sofía, adoptó dos textos complementarios: la Carta del Turismo y el Código Ético Mundial para el Turismo. Como explica García Palacios, «en el primero se especificaban los deberes de los Estados para con los turistas y los derechos de las comunidades receptoras, a las que los Estados deben proteger y hacer cumplir sus derechos» (García Palacios, 2016: 602).

En el marco teórico, el término turismo comunitario aparece por primera vez en la obra de Murphy (1985). No obstante, esta modalidad de turismo todavía tardaría algunos años en establecerse. Durante la década de los noventa surgen a lo largo del globo, y de manera muy significativa en Ecuador —país líder en TC (Hutchins, 2007)-, distintos emprendimientos de TC. Es precisamente en Ecuador, en la ciudad de Otavalo - una ciudad de marcado carácter indígena- donde tiene lugar el Encuentro Técnico Internacional sobre Gestión del Turismo Sostenible y Competitivo: Alianzas entre Estado, Empresa y Comunidad, realizado durante el año 2001; un encuentro que daría como resultado final una importante declaración sobre el TC (OIT, 2001). Este encuen- 
tro marca la formalización del TC y pone las bases para la formación de distintas instituciones, gubernamentales y no gubernamentales, públicas y privadas, relacionadas con el TC.

El TC se ha definido de diversas maneras. El Fondo Mundial para la Naturaleza (WWF) define el TC como «aquel tipo de turismo en el que la comunidad local tiene un control sustancial, participa en su desarrollo y manejo, y una importante proporción de los beneficios se quedan en la comunidad» (WWF, 2001: 4). Una definición algo laxa, pero, precisamente por ello, menos problemática es la de Gascón y Cañada, para quienes el TC se caracteriza por ser un «turismo de pequeño formato, establecido en zonas rurales y en el que la población local, a través de sus estructuras organizativas, ejerce un papel significativo en su control y gestión» (Gascón y Cañada, 2005: 159). Por su parte, Ortega y Vicente centran su definición del TC en el hecho de que pequeñas comunidades rurales abran sus hogares a los turistas, permitiendo conocer sus costumbres y su vida cotidiana. En esta forma de turismo los miembros de la comunidad asumen distintos roles: guías, hospedadores, cocineros o artesanos (Ortega y Vicente, 2013: 10).

En definitiva, se puede ver cómo el TC es un fenómeno «multidimensional e híbrido» (Ruiz-Ballesteros y Hernández-Ramírez, 2010), en el cual se encuentran casos más cercanos al turismo tradicional, con empresas privadas funcionando a modo de agencias turísticas; pero también con emprendimientos autónomos en los que las comunidades tienen un mayor protagonismo (Wunder, 200o). En todo caso, lo que caracteriza de manera general a esta propuesta de TC es la participación activa de una comunidad indígena —en ocasiones mestiza-, que ofrece como producto turístico su propia comunidad, así como ocasionalmente servicios de alojamiento, restauración y venta de artesanías, entre otros.

Los beneficios del TC han sido largamente destacados por la importante bibliografía existente sobre el tema. El TC sería una forma particular de turismo que permitiría la supervivencia, pero también el «empoderamiento de las comunidades» (Hiwasaki, 2006: 677). Este tipo de turismo se caracterizaría por ser responsable, solidario y ecológico; al mismo tiempo que ayudaría a reivindicar y promocionar los «valores culturales de los sectores rurales» (RuizBallesteros y Solis Carrión, 2007: 195). De este modo, el TC supondría el gran beneficio de presentar una «relación desde la interculturalidad» (Oyarzún, Estrella, Capiran y Vazquez, 2010: 8).

Una reivindicación recurrente entre los defensores del TC es que este tipo de turismo no se limita a producir beneficios económicos, sino que también 
supone una revalorización de la cultura, una recuperación de los productos nativos y una protección de los recursos naturales. Así lo expresaba la declaración que quedó como conclusión del mencionado Encuentro Técnico Internacional sobre Gestión del Turismo Sostenible y Competitivo: Alianzas entre Estado, Empresa y Comunidad, según la cual el turismo comunitario debe «promover las expresiones genuinas de la identidad cultural de los pueblos indígenas, sus valores, símbolos y costumbres, los mismos que constituyen una fuente de diferenciación y competitividad para sus productos turísticos» (OIT, 2001: 1). Como veremos a continuación, todas estas pretensiones, de índole económica, cultural y comunitaria, son difícilmente conciliables. Desde la literatura propia del TC, así como de la rama conocida como indigenous ethics, intentaremos mostrar algunos de los problemas relacionados con estas cuestiones.

\section{PROBLEMAS ÉTICO-ANTROPOLÓGICOS DEL TURISMO COMUNITARIO}

Como acabamos de exponer, el TC suele presentarse como una propuesta no solo económicamente rentable, sino respetuosa con la propia población de las comunidades. Además de la generación de empleos y el aumento de renta, se afirma que el turismo comunitario contribuye al cuidado del medio ambiente y a que la población indígena conserve sus territorios. Sin negar frontalmente estas afirmaciones, vamos a tratar de problematizar cierta apreciación, a nuestro modo de ver excesivamente benigna, del TC. Por supuesto, existe una literatura importante sobre el tema en relación a la ética del turismo en un sentido amplio (Jenkins, 2002; Blázquez y Cañada, 2011; Fennell, 2018); pero aquí nos gustaría abordar concretamente los problemas éticos específicos suscitados por el TC.

En primer lugar, algunos autores han puesto en cuestión la repercusión «mínima o nula» (García Palacios, 2016: 621) que el TC realmente genera en las comunidades, así como la modesta mejora de ingresos que perciben las familias, una mejora que está muy lejos de erradicar la pobreza de la población local; punto que, sin embargo, es muchas veces defendido como gran valor del TC (Deller, 2010). Unos ingresos que, además, en muchos casos dependen mayoritariamente de ayudas externas (Manyara, Jones y Botterill, 2006; Kiss, 2004), lo cual crea una peligrosa dependencia para las comunidades. En este sentido, también resulta conflictivo que una gran parte de los emprendimien- 
tos de TC se originen por iniciativa de agentes externos, en lugar de surgir desde las propias comunidades (Paniagua, 2002).

Tampoco la aportación del TC a la conservación ambiental parece indiscutible: si bien otros tipos de turismo tradicionales son más agresivos con el medio ambiente, el TC también suele implicar el uso de transportes contaminantes, además del lógico incremento de habitantes temporales que el turismo trae consigo. Más allá del grado en que podamos considerar al TC como una ayuda a la conservación del medio ambiente, queda también la duda de si el TC no constituye, de manera similar al ecoturismo, una mercantilización de la naturaleza (Duffy, 2008), que también podría ser reprobable en términos éticos. Asimismo, el TC conlleva, en ciertas ocasiones, que ciertas actividades tradicionales queden olvidadas o en un segundo plano debido a la necesidad de centrarse en la atención turística (Vargas-Cumbajín et al., 2017: 84).

En todo caso, y aun si aceptáramos todas las virtudes antes mentadas, debemos ser conscientes de algunos problemas específicos que el TC trae consigo. Problemas que, como vamos a ver, no son meras desviaciones corregibles de un sistema en esencia aceptable, sino que acaban siendo problemas radicales y constitutivos de la propuesta de TC. La cuestión fundamental que discutiremos a continuación es si el TC no supondría la descomposición y, en último término, la destrucción de la comunidad como tal, y su sustitución por una ficción alienadora que profundiza en alguno de los ya graves problemas de la población indígena que suele regentar estos emprendimientos.

Este punto ya ha sido destacado por algunos intérpretes, como Cabanilla, quien afirma que «el acelerado proceso de implantación en el territorio del turismo comunitario ha permitido una rápida mercantilización de varios elementos de vida socio-cultural de las comunidades indígenas» (Cabanilla, 2015: 362). Esta mercantilización, argumenta este autor, conlleva una adaptación, formal y de fondo, de parte de la comunidad, generando una hiperrealidad que amenaza seriamente la integridad cultural de la comunidad (Cabanilla, 2015: 356). Esta adaptación y turistificación de la comunidad, que muchas veces se presenta como inocua y exenta de problemas, es, sin embargo, extremadamente preocupante en diversos sentidos. Por un lado, la cultura autóctona pasa a depender, en cierto modo, de la cultura externa de los turistas, que pasan a erigirse en validadores de dicha cultura en base a sus patrones externos (Urry, 1990). Como explica Grünewald (2002: 1009):

La búsqueda de lo exótico se está convirtiendo en un proceso autodestructivo debido a la abrumadora influencia del observador sobre lo observado. En otras palabras, la misma 
presencia del turista haría al nativo menos exótico y tradicional, transformándolo en un «touree», o bien un actor que modifica su comportamiento en función de la percepción de lo que es atractivo para el turista.

La mercantilización suele conceptualizarse como el proceso por el cual algo pasa a situarse en el contexto del intercambio de bienes y pasa a ser evaluado en términos de su valor de cambio, convirtiéndose en un producto (Cohen 1988). Es difícil pensar que el TC no produce este mismo efecto en una comunidad que pasa a ser, en sus diferentes aspectos, un bien de consumo. Como explican Ruiz-Ballesteros y Hernández-Ramírez (2010: 211-2012):

En el proceso de mercantilización se percibe una síntesis de la «mirada del turista» (Urry, 1990), la «autenticidad escenificada» (MacCannell, 1976) y la «etnicidad reconstruida» (MacCannell, 1992). En general, las comunidades indígenas buscan presentarse de la forma en que los turistas los perciben para poder venderse mejor; la mirada del turista termina por dar forma a la construcción de ellos mismos. Los rasgos, objetos, elementos y prácticas de su vida cotidiana se convierten en productos turísticos, se «mercantilizan».

El turismo trastoca el funcionamiento cotidiano y natural de la comunidad, eliminando cualquier posibilidad de autenticidad e instaurando una dinámica impostada que tiene al turista como núcleo, en lugar de ser los integrantes de la comunidad el centro de la misma. Esto trae consigo una «banalización de los ritos mágico-religiosos» (Cabanilla, 2015: 362), que también pasan a ser un evento turístico desarrollado por actores que, inevitablemente, van contra la lógica interna comunitaria, la cual pasa a regirse por los criterios ajenos de los turistas. Incluso las prácticas menos solemnes, como puede ser la cocina o la elaboración de artesanías, pasan a ser producciones y exhibiciones cuyo propósito no tiene que ver con el sustento de la comunidad, sino con la satisfacción del turista consumidor.

Los roles tradicionales de la comunidad y las relaciones entre sus miembros también quedan irremediablemente trastocados por el TC. La comunidad pasa a girar y funcionar en torno al turista: el pastor debe realizar sus tareas de modo que sea visible y vistoso para el turista; el campesino debe presentar y tener presentable su campo para el turista; los niños no pueden realizar juegos que entorpezcan la visita de los turistas, etc. Incluso algunas de las supuestas ventajas que en este sentido el TC aparenta proporcionar se muestran como problemáticas. Pues, si bien en ocasiones se afirma que el TC mejora la autoestima de la mujer y contribuye a su independencia, lo cierto es que también «puede acabar consolidando sus roles tradicionales» (Vargas-Cumbajín et al., 
2017: 84) al obligarla a dedicarse a tareas domésticas como la cocina y el alojamiento de los visitantes (Henrici, 2007).

\section{UNA DIFICULTAD AÑADIDA. LA DESATENCIÓN A LAS INDIGENOUS ETHICS}

Otra fuente de problemas relativamente poco atendida en la literatura sobre el TC es el campo de las indigenous ethics (Tassell, Herbert, Evans y Young, 2012; Kelley, Belcourt-Dittloff, Belcourt y Belcourt, 2013). Con este epíteto nos referimos a la ética específica del trato con poblaciones indígenas, contexto en el cual habitualmente surgen problemas de desequilibrios de poder, así como de inconmensurabilidad cultural. La propia implantación del TC supone en sí misma una negación de estas cuestiones, por cuanto entiende que la relación entre los turistas extranjeros y la población indígena se da en unos términos de naturalidad y normalidad que no demanda consideraciones previas de este tipo. Sin embargo, como trataremos de mostrar, esto está lejos de ser el caso.

Un primer punto a tener en cuenta es el problema antes mencionado de la autenticidad escenificada. Como explica MacCannell, hay un serio peligro de que la etnicidad quede transformada en una «etnicidad-para-el-turismo en el que las culturas exóticas figuran como atracciones estrella» (MacCannell, 1992: 158). En este contexto, los nativos pueden sentirse impelidos a «volverse nativos-para-los-turistas» (MacCannell, 1992: 159). Desde este punto de vista, el TC podría constituir un ataque todavía más pernicioso sobre la etnicidad al suplantarla con un constructo impostado y basado en las exigencias externas de los turistas.

Como explican Kelley y sus colaboradores, «la colonización amenazó la identidad, la cultura, las creencias religiosas y las opiniones epistemológicas de los pueblos indígenas» (Kelley, Belcourt-Dittloff, Belcourt y Belcourt, 2013: 2146). Esta problemática llega hasta nuestros días, alcanzando ámbitos como el TC que, de manera inadvertida, pueden traer consigo discriminaciones o prácticas abusivas respecto de la comunidad indígena. Es imprescindible, por tanto, revisar fundamentalmente cualquier interacción con las comunidades indígenas que pueda derivar en situaciones problemáticas de este tipo (Kelley, Belcourt-Dittloff, Belcourt y Belcourt, 2013: 2150).

En las relaciones entre turistas y poblaciones indígenas siempre se debe tener en cuenta la posible existencia de diferenciales de poder, especialmente 
en el caso de que esas relaciones estén diseñadas y planificadas desde la perspectiva de las poblaciones externas, habitualmente de pasado colonial (Tassell, Herbert, Evans y Young, 2012: 1). Es necesario tener presente un pasado de dominación que puede operar como base a ciertos abusos; abusos que la propia comunidad puede aceptar explícitamente, pero que, podría argumentarse, reproducen ciertas relaciones de poder pasadas y rechazables.

Es también crucial tener en cuenta, como anticipábamos un poco antes, las cosmovisiones y los modos de vida indígenas, que, en muchos casos, no coinciden con algunos principios occidentales relativos al bienestar, la privacidad o el consentimiento (Tassell, Herbert, Evans y Young, 2012: 1). Respecto de la privacidad, por ejemplo, habría que considerar seriamente si el concepto occidental de lo privado es aplicable a comunidades donde lo común se entiende en un sentido mucho más amplio, integral y cotidiano de lo que se entiende en las sociedades occidentales, donde lo común prácticamente equivale al Estado (Tassell, Herbert, Evans y Young, 2012: 4). Como explica Smith, «el individuo, como unidad social básica de la que proceden otras organizaciones y relaciones sociales, es otro sistema de ideas que debe ser entendido como parte de la impronta de Occidente» (Smith, 1999: 49).

Asumir que el TC está cumpliendo con los estándares éticos de otros tipos de actividades comerciales porque se están respetando los derechos individuales de sus integrantes podría no ser suficiente desde la lógica comunitaria. Tomando en cuenta esta advertencia, podría considerarse que el turista participante del TC está, pese a que no haya ninguna queja explícita por parte de los habitantes, violentando la privacidad de la comunidad. Para los integrantes de la comunidad puede que no exista lo privado en los términos típicos occidentales, pero para los turistas sí existe tal concepto, lo que conlleva, o podría considerarse que conlleva, una trasgresión por parte de los turistas.

En este sentido, es muy plausible imaginar que los miembros de la comunidad, al entender su identidad como directa e insoslayablemente unida y subordinada a la de su comunidad, acepten el TC por defecto. Es claro, por último, que, para las culturas indígenas, el compartir, la responsabilidad colectiva y la propiedad colectiva son modos de pensar y hacer las cosas que caracterizan su modo de vida (Ermine et al., 2004). Cuando estos principios son aprovechados por actividades comerciales como el TC, resulta comprensible considerar que estamos ante una actividad problemática desde el punto de vista ético.

Sería, por tanto, un error asumir que el consentimiento general para implantar el TC en una comunidad implique una verdadera aceptación, conscien- 
te e integral, del mismo por cada miembro de la comunidad. En otros ámbitos, como es el caso de la atención sanitaria y más específicamente psicológica, esta asunción del consentimiento ha resultado muy problemática. Algunos autores han intentado establecer procedimientos que prevengan este tipo de problemas. Una propuesta concreta es la de Kelley y sus colaboradores, que, entre otros puntos, establece la necesidad de: «(3) una resolución firmada por todas las tribus involucradas, $(4)$ cartas de apoyo firmadas por individuos [...]» para concluir solicitando «copias de los procedimientos y formularios de consentimiento informado» (Kelley, Belcourt-Dittloff, Belcourt y Belcourt, 2013: 2150). Sin embargo, el sentido y la efectividad de estos procedimientos ofrece muchas dudas cuando se piensa desde las características propias y específicas de la comunidad. Este tipo de salvaguardas legales supone caer en la lógica occidental contractualista; pero el contractualismo es, en cierto modo, el acta de defunción de la comunidad.

\section{TURISMO Y COMUNIDAD, ¿ES POSIBLE LA COEXISTENCIA?}

Como se ha apuntado, el problema ético fundamental que presenta el TC es precisamente el hecho de usar la comunidad como elemento turístico. Diversos autores han denunciado, en términos globales y epocales, este ataque a la comunidad por parte del mundo moderno. Bauman es uno de los pensadores que más ha reflexionado sobre este punto, acuñando su concepto de modernidad líquida. La liquidez a la que este autor se refiere tiene que ver con el «derretimiento de los sólidos», es decir, «la "profanación de lo sagrado": la desautorización y la negación del pasado, y primordialmente de la "tradición"» (Bauman, 2000: 9). Entre los elementos constituyentes de la tradición que la modernidad viene a arrasar, la comunidad aparece en un lugar primordial. La modernidad destruye desde la raíz los lazos comunitarios de las sociedades tradicionales, atomizando a sus miembros y creando una cultura intrínsecamente individualista. Ni la propuesta baumaniana, ni el trabajo presente, se aferran a una comunidad idealizada. Este análisis no opera bajo una idea esencialista de la comunidad. Pero eso no obsta para reconocer la ruptura que la modernidad trae consigo en lo referente a la comprensión y, más fundamentalmente, la vida de la comunidad.

La modernidad erige a la economía en realidad focal, convirtiendo todo en mercancía y diluyendo cualquier otra realidad humana en su comercialidad. Como explica J. Fuentes, la modernidad y su reduccionismo económico 
ponen en marcha «la disolución antropológica del mundo, es decir, la disolución del sentido de la vida humana misma (en cuanto que comunitaria) en el mundo» (Fuentes, 2013: 6). Una reflexión que este mismo autor amplía al afirmar que:

[...] todo el horizonte «moral» (por llamarlo de algún modo) de estas neosocialdemocracias viene a reducirse a la construcción estatal de unas sociedades nacionales de acomodados consumidores satisfechos que por ello mismo se encuentren los más abstraídos o desligados posible de todo vínculo personal y comunitario tradicional. De ahí su característico empeño compulsivo por llevar a cabo una política cultural intensiva de disolución, desde el Estado, de los vínculos comunitarios y personales tradicionales solidaria con el desarrollo de semejante tipo de bienestar, una disolución esta que es justamente revestida por la ideología de una presunta emancipación felicitaria de dichos vínculos, que sin duda son ideológicamente despreciados y estatalmente perseguidos como lastres reaccionarios del pasado. Y de aquí sin duda la atmósfera moralmente hedionda que inexorablemente se respira en el ámbito de las actuales socialdemocracias, así como en sus inevitables alrededores, esto es, en aquellas ideologías emancipatorias (Fuentes, 2013: 11).

El TC supondría, desde esta perspectiva, un intento de corromper aquellos últimos reductos donde la modernidad aniquiladora de lo comunitario no había llegado todavía. Es precisamente en aquellas comunidades, predominantemente de población indígena, impermeables durante mucho tiempo a la lógica moderna economicista e individualista, donde el TC se infiltra y amenaza trastocar sus prácticas y cotidianeidad. Es precisamente esta globalización económica la que, según autores como MacCannell, está dando lugar a unas determinadas formas de etnicidad reconstruida: «las nuevas etnicidades reconstruidas están apareciendo como el resultado más o menos automático de la entrada de todos los grupos del mundo en una red global de transacciones comerciales» (MacCannell, 1992: 168). Esta etnicidad reconstruida ya no sería, claro está, una auténtica etnicidad. Y la comunidad asociada a esa identidad y tradición indígena sería, por lo mismo, muy distinta a la reconstrucción que la modernidad economicista impone.

Algunos autores, no obstante, han rebatido este punto de manera notable. Stronza (2001) y Ryan (2005) han destacado, por separado, la peculiar idiosincrasia del TC, distinguiéndolo, al menos preliminarmente, del turismo convencional. Ruiz-Ballesteros y sus colaboradores han desarrollado un análisis de lo que denominan como community-based tourism ('turismo basado en la comunidad') que cuestiona la realidad de los problemas morales antes aducidos. Para estos autores, frente a lo expuesto anteriormente, «la actividad turística trae 
consigo una ampliación de los intereses comunes» (Ruiz-Ballesteros et al., 2008: 408), y esta ampliación no comporta un daño esencial a la propia comunidad. Estos autores son conscientes de los riesgos que el TC trae consigo de convertir a la comunidad y su funcionamiento en un simulacro, pero piensan que «el contexto comunitario en el que tiene lugar los puede minimizar notablemente en virtud de su encarnación del mercado» (Ruiz-Ballesteros et al., 2008: 412). Este punto queda condensado en el siguiente párrafo:

La diferencia fundamental con otros sectores económicos es que el turismo desarrollado comunitariamente permite que la comunidad en vez de diluirse en el Mercado-Estado en el que se asienta, se reafirme en virtud de sus márgenes de control sobre la actividad turística, del que además no depende en exclusividad. Por otra parte, al hacerse necesario más que nunca lo propio, lo comunitario, la actividad turística fortalece al grupo, no solo en el sentido de rentabilizar las estructuras políticas y las prácticas comunitarias, sino en el de la valoración y el redescubrimiento de lo comunitario e indígena. (Ruiz-Ballesteros et al., 2008: 414)

Ruiz-Ballesteros y Hernández-Ramírez (2010) insisten sobre este punto en otro texto. Allí estos autores contraponen actividades de intercambio capitalista — como pueden ser las relacionadas con la ganadería, pesca, etc. - con el TC, en el cual «paradójicamente, las comunidades pueden ejercer un mayor grado de control sobre la actividad (su producción y comercialización)» (RuizBallesteros y Hernández-Ramírez, 2010: 211). Estos autores, apoyándose en García Canclini (1995) y Ortiz (2004), disputan la visión generalmente negativa que se tiene del mercado, explicando que este también puede ser un lugar para la construcción personal y las relaciones significativas (Ruiz-Ballesteros y Hernández-Ramírez, 2010: 211). Como acaban expresando estos dos autores, «el mercado se convierte en una oportunidad para la comunidad, mientras que la comunidad es una oportunidad para el mercado» (Ruiz-Ballesteros y Hernández-Ramírez, 2010: 224).

Sin embargo, y pese a que las matizaciones de estos autores deben ser tenidas en cuenta, el punto fundamental de nuestra crítica anterior se mantiene, pues el núcleo de los problemas éticos del TC no tiene que ver con cuestiones de índole práctico, como la capacidad de la comunidad para preservar el control de la actividad económica derivada del TC, sino con el carácter que la unión comunitaria toma cuando está atravesada por el turismo y la esfera comercial. La comunidad generada a partir del TC, los lazos comunitarios y el tipo de colectividad creado por el TC son, desde nuestra perspectiva, una ruptura de lo que en su origen era la auténtica comunidad. Por mucho que el TC 
se presente como un fenómeno «empoderador» (Wallace y Russell, 2004: 237), el poder que la comunidad gana se logra al precio de su evaporación como tal comunidad.

Esto se ve con mayor claridad con uno de los aspectos destacados por diversos teóricos y por algunos emprendimientos de TC: la propuesta de un turismo convivencial. Esta modalidad de turismo comunitario iría un paso más allá y ofrecería a los turistas la experiencia de convivir con los miembros de la comunidad. La comunidad se abriría plenamente para acoger en su seno a los turistas y desarrollar sus quehaceres cotidianos junto a ellos. De este modo, «los hombres y mujeres, sus hijos, sus casas, la manera en la que sufren, celebran y, en resumen, viven y ven el mundo, son de hecho el producto» (RuizBallesteros y Hernández-Ramírez, 2010: 208). Ruiz-Ballesteros y sus colaboradores consideran que «el TC se desarrolla y consolida en tanto en cuanto tiene un encaje lógico en el ethos de las comunidades: cuando aprovecha su urdimbre de relaciones y su capacidad de improvisación» (Ruiz-Ballesteros et al., 20o8: 413).

Mas aquí lo problemático es precisamente eso: el aprovechamiento, la conversión en producto comercial de la convivencia, de ese núcleo vivo de la comunidad. Es simplemente imposible que la comunidad se mantenga en su naturalidad cuando se ve obligada a incluir los elementos inevitablemente extraños que son los turistas. El turismo introduce una anomalía e interrupción insuperable de lo cotidiano. Y esto es así porque no puede haber turismo sobre la vida cotidiana, no puede haber turismo y convivencia. En el momento en que la lógica del turismo se infiltra en lo cotidiano, lo cotidiano deja de ser tal y la convivencia, caracterizada por la confianza, la seguridad y la normalidad, queda irrevocablemente anulada. Por no hablar de lo conflictivo que puede resultar pensar en el carácter de exposición casi zoológica en el que se cae en esta modalidad de turismo convivencial (y, por extensión, en toda forma de $\mathrm{TC})$.

\section{CONCLUSIONES}

En conclusión, el TC trae consigo toda una serie de problemas éticos difíciles de superar. Algunos de estos problemas, como el cuidado del medio ambiente, el control por parte de agentes externos o el diferencial de poder existente entre turistas y población indígena podrían ser parcialmente corregidos. $\mathrm{O}$, al menos, podrían ponerse en una balanza frente a algunas de las 
virtudes justamente expuestas por los defensores del TC. No obstante, y sin negar lo anterior, debemos afirmar que el TC lleva inscrito en su misma naturaleza el problema nuclear al que nos hemos venido refiriendo a lo largo del texto. El TC, independientemente de sus propuestas concretas, implica la disolución de la comunidad en tanto que comunidad. Una vez que la lógica del turismo se introduce en la comunidad, no se puede decir que esta siga siendo lo que era hasta entonces.

Por supuesto, no podemos aquí aducir ningún concepto esencialista de comunidad. La comunidad no es un factum, sino un faciendum, una realidad en marcha que debe renovarse cada día desde la cotidianeidad y la convivencia. Ruiz-Ballesteros y sus colaboradores apuntan, matizadamente, a que «la comunidad precisa ser desmitificada pero asimismo hemos de reconocer su evidente existencia y funcionalidad» (Ruiz-Ballesteros et al., 2008: 401). No obstante, el punto central, tan difícil de entender desde nuestra perspectiva occidental, es que la funcionalidad de la comunidad es ser la base, la condición de posibilidad, de un determinado tipo de vida. Decir esto no implica ninguna mitificación ni esencialismo. Es el simple reconocimiento de una realidad, la comunidad, que, si bien es para la mayoría de occidentales ajena y desconocida, constituye el fundamento de un determinado tipo de vida. Lo que aquí argumentamos es que este revivir y rehacerse diario de la comunidad, su propia conformación dinámica en tanto que realidad, queda gravemente cortocircuitado por la introducción de ese elemento extraño que es el turismo. Como explica Smith desde su propia experiencia e investigaciones, las comunidades refieren una y otra vez la dificultad de seguir siendo indígenas tras el contacto occidental (Smith, 1999: 197-199).

Se puede argumentar, no obstante, que la vida comunitaria está en vías de extinción y que la suave disolución llevada a cabo por el TC es mucho mejor que otros tipos de destrucción violenta. Implementar el TC en una comunidad podría ser visto como una suerte de eutanasia comunitaria. Es posible que, en términos prácticos, el TC sea la menos mala de las alternativas. Pero, si este es el caso, hay que ser conscientes, no obstante, de los problemas éticos a los que venimos haciendo alusión.

Por todo lo expuesto, parece difícil postular alternativas éticas para el TC. Podrían darse pasos en el camino de concienciar a los integrantes de las comunidades del peligro que supone la implantación del TC para sus costumbres y formas de vida. Mas, como se ha visto, suelen ser las propias comunidades las que abrazan el TC y lo entienden como una forma de reafirmar y reforzar su 
cultura e identidad. Probablemente no haya una manera efectiva de parar una mercantilización de la vida humana que constituye el signo de los tiempos.

Una propuesta concreta consistiría en llevar a cabo el equivalente a encuestas de satisfacción en la comunidad, preguntando si la implantación del TC ha sido realmente benéfica. Estas encuestas o, si fuera posible, entrevistas personalizadas deberían ser muy sutiles y minuciosas, intentando captar el verdadero sentir de los miembros de la comunidad. Esto podría ayudar a las comunidades a evaluar y reevaluar su adopción del TC. Sin embargo, como decimos, es difícil que estas prácticas —que no dejan de inscribirse en la lógica comercial moderna- puedan realmente prevenir el trastocamiento producido por el TC en la comunidad.

Podemos, en todo caso y como programa de mínimos, intentar que el TC se haga más consciente de los problemas antes expuestos, como los relativos a la injerencia de agentes externos o los relacionados con el trato específico con personas indígenas, con los desequilibrios de poder y los problemas de inconmensurabilidad cultural que aparecen en estos contextos. En un mundo global donde incluso las más pequeñas y apartadas comunidades se ven sujetas a las lógicas del mundo moderno, puede que este tipo de propuestas de mínimos sea lo máximo a lo que podemos aspirar.

\section{BIBLIOGRAFÍA}

Bauman, Zygmunt (200o). Modernidad líquida. Buenos Aires: Fondo de Cultura Económica.

Blázquez, Macia y Cañada, Ernest (eds.) (2011). Turismo Placebo. Nueva colonización turística: del Mediterráneo a Mesoamérica y El Caribe. Lógicas espaciales del capital turístico. Managua: Edisa. Burns, Peter M. y Holden, Andrew (1995). Tourism: A New Perspective. London: Prentice Hall.

Cabanilla, Enrique (2015). Impactos culturales del turismo comunitario en Ecuador sobre el rol del Chamán y los ritos mágico-religiosos. Estudios y Perspectivas en Turismo, 24(2), 356373. 
Cohen, Erik (1988). Authenticity and Commoditization in Tourism. Annals of Tourism Research, 15, 371-386.

Deller, Steven (2010). Rural poverty, tourism and spatial heterogeneity. Annals of Tourism Research, 37(1), 180-205.

Duffy, Rosaleen (2008). Neoliberalising Nature: Global Networks and Ecotourism Development in Madagascar. Journal of Sustainable Tourism, 16(3), 327-344.

Ermine, Willie, Sinclair, Raven y Jeffrey, Bonnie (2004). The ethics of research involving indigenous peoples: Report of the Indigenous Peoples' Health Research Centre to the Interagency Advisory Panel on Research Ethics. Regina, SK: Indigenous Peoples' Health Research Centre.

Fennell, David A. (2008). Ecotourism. London, New York: Routledge. Fennell, David A. (2018). Tourism Ethics. Clevedon: Channel View Publications.

Fuentes Ortega, Juan Bautista (2013). Entrevista: Política, metapolítica y modernidad. El caso de España. Con una adenda sobre la idea de Ortega de la «crisis del hombre europeo». E-print UCM.

García Canclini, Néstor (1995). Consumidores y ciudadanos. México: Grijalbo.

García Palacios, Carlos (2016). Turismo comunitario en Ecuador: ¿Quo vadis? Estudios y Perspectivas en Turismo, 25(4), 597-614.

Gascón, Jordi y Cañada, Ernest (2005). Viajar a todo tren: turismo, desarrollo y sostenibilidad. Barcelona: Icaria.

Grünewald, Rodrigo de Azeredo (2002). Tourism and cultural revival. Annals of Tourism Research, 29(4), 1004-1021.

Hampton, Mark P. (2003). Entry points for local tourism in developing countries: evidence from Yogyakarta, Indonesia. Geografiska Annaler, 85(2), 85-101.

Henrici, Jane (2007). Género, turismo y exportación: ¿llamando a la plata en el Perú? Anthropologica, 25(25), 83-101. 
Hiwasaki, Lisa (2006) Community-based tourism: A pathway to sustainability for Japan's protected areas. Society and Natural Resources, $19(8), 675^{-692 .}$

Hutchins, Frank (2007). Footprints in the Forest: Ecotourism and Altered Meanings in Ecuador's Upper Amazon. Journal of Latin American and Caribbean Anthropology, 12(1), 75-103.

Jenkins, Tiffany (2002). Ethical tourism: Who benefits? London: Hodder \& Stoughton.

Kelley, Allyson, Belcourt-Dittloff, Annie, Belcourt, Cheryl y Belcourt, Gordon (2013). Research ethics and indigenous communities. American Journal of Public Health, 103(12), 2146-2152.

Kiss, Agnes (2004). Is community-based ecotourism a good use of biodiversity conservation funds? Trends in Ecology and Evolution, 18, 222-237.

MacCannell, Dean (1976). The Tourist: A New Theory of the Leisure Class. New York: Schocken Books.

MacCannell, Dean (1992). Reconstructed Ethnicity: Tourism and Cultural Identity in Third World Communities. En MacCannell, Dean. Empty meeting grounds: the tourist papers. London: Routledge. Manyara, Geoffrey, Jones, Eleri y Botterill, David (2006). Tourism and Poverty Alleviation: The Case for Indigenous Enterprise Development in Kenya. Tourism, Culture and Communication, 7(1), 19-37.

Mowforth, Martin y Munt, Ian (1998). Tourism and Sustainability:

New Tourism in the Third World. New York: Routledge.

Murphy, Peter E. (1985) Tourism: A community approach. London: Methuen.

Organización Internacional del Trabajo - OIT (2001). Declaración de

Otavalo sobre el turismo comunitario. Ginebra.

Organización Mundial del Turismo - OMT (1985). Carta del turismo y código del turista. Sofía. 
Ortega, Luis y Vicente, Geoconda (2013). Plan de Desarrollo Turístico Comunitario en la Parroquia de Toacaso. Latacunga, Ecuador: Universidad Central del Ecuador.

Ortiz, Santiago (2004). Cotacachi: una propuesta por la democracia participativa. Quito: FLACSO.

Oyarzún, Edgardo, Estrella, Mateo, Caripan, Héctor y Vazquez, Edmundo (2010). Manual de Turismo Comunitario. Cuenca, Ecuador: Copifull.

Paniagua, Ángel (2002). Urban-rural migration, tourism entrepreneurs and rural restructurin in Spain. Tourism Geographies, 4(4), 349-371.

Ruiz-Ballesteros, Esteban y Solis Carrión, Doris (2007). Turismo Comunitario en el Ecuador. Desarrollo y sostenibilidad social. Ecuador: Abya-Yala.

Ruiz-Ballesteros, Esteban, Hernández Ramírez, Macarena, Coca Pérez, Agustín, Cantero, Pedro A. y Campo Tejedor, Alberto del (2008). Turismo comunitario en Ecuador: Comprendiendo el community-based tourism desde la comunidad. Pasos: Revista de Turismo y Patrimonio Cultural, 6(3), 399-418.

Ruiz-Ballesteros, Esteban y Hernández-Ramírez, Macarena (2010). Tourism that Empowers? Commodification and Appropriation in Ecuador's Turismo Comunitario. Critique of Anthropology, 30(2), 201-229.

Ryan, Chris (2005). Tourist - Host Nexus - Research Considerations. En Ryan, Chris y Aicken, Michelle (Eds.). Indigenous Tourism: The Commodification and Management of Culture (1-11). Amsterdam: Elsevier.

Smith, Linda Tuhiwai (1999). Decolonizing Methodologies: Research and Indigenous Peoples. London: Zed Books.

Stronza, Amanda (2001). Anthropology of Tourism: Forging New Ground for Ecotourism and Other Alternatives, Annual Review of Anthropology, 30, 261-283. 
Tassell, Natasha, Herbert, Averil, Evans, Ian y Young, Patricia (2012). Principles of Indigenous Ethics and Psychological Interventions. The Oxford Handbook of International Psychological Ethics. Oxford: Oxford Press.

Urry, John (1990). The tourist gaze: Leisure and travel in contemporary societies. London: Sage Publications.

Vargas-Cumbajín, Carlos A., Yánez-Segovia, Sebastián G., Hernández-Benalcázar, Herman W., Méndez-Játiva, Jhony F., Valdiviezo-Leroux, Washington R. y Tafur, Valdano (2017). La situación del turismo comunitario en Ecuador. Dominio de las ciencias, $4(1)$, 80-101.

Wallace, Gillian y Russell, Andrew (2004). Eco-cultural Tourism as a Means for the Sustainable Development of Culturally Marginal and Environmentally Sensitive Regions. Tourist Studies, 4(3), 235254 .

Wunder, Sven (200o). Ecotourism and economic incentives - an empirical approach. Ecological economics, 32, 465-479.

WWF International (2001). Guidelines for community-based ecotourism development WWF International. London: WWF International. 